\title{
White blood cells levels and PCOS: direct and indirect relationship with obesity and insulin resistance, but not with hyperandogenemia
}

\author{
Olga Papalou, ${ }^{1}$ Sarantis Livadas, ${ }^{1}$ Athanasios Karachalios, ${ }^{1}$ Nikoleta Tolia, ${ }^{1}$ \\ Panayiotis Kokkoris, ${ }^{2}$ Konstantinos Tripolitakis, ${ }^{2}$ Evanthia Diamanti-Kandarakis ${ }^{1}$
}

${ }^{1}$ Endocrine Unit, Third Department of Internal Medicine, Medical School, National and Kapodistrian University of Athens, ${ }^{2}$ Department of Endocrinology \& Diabetes, 251 Hellenic Air Force \& VA General Hospital, Athens, Greece

\begin{abstract}
OBJECTIVE: To study white blood cells count (WBC) in women suffering from PCOS and compare these results with age and BMI-matched healthy women. The specific aim of this study was to assess the possible correlations of $\mathrm{WBC}$ with the major components of PCOS, obesity, insulin resistance and hyperandrogenism. DESIGN: Anthropometrical, metabolic and hormonal data were analyzed from 203 women with PCOS (NIH criteria) and 76 agematched controls. RESULTS: In the total population studied $(\mathrm{N}=279)$, WBC was significantly higher $(P=0.003)$ in the PCOS group compared with age-matched healthy women and was positively correlated with $B M I(r=0.461, p<0.001)$, total testosterone $(r=0.210, p<0.001)$, insulin $(r=0.271, p<0.001)$, triglycerides $(r=0.285, p<0.001)$, HOMA score $(r=0.206, p=0.001)$, FAI $(\mathrm{r}=0.329, \mathrm{p}<0.001)$ and negatively correlated with SHBG $(\mathrm{r}=\mathbf{- 0 . 3 0 0 ,}, \mathrm{p}<0.001)$ and HDL $(\mathrm{r}=$ $\mathbf{- 0 . 2 2 2}, \mathbf{p}<0.001)$. Due to the fact that WHR was only available in the group of PCOS women, the role of central adiposity is assessed only in this group. Multiple regression analysis in the PCOS group, including WHR, revealed BMI, SHBG and TGL as the main predicting factors of WBC. Multinomial logistic regression analysis was also conducted and overweight/obesity was the sole independent risk factor for elevated WBC (higher tertile) (OR:0.907 CI:0.85-0.96, $p=0.002)$. After dividing the sample based on BMI in the lean subgroups, WBC did not differ significantly between PCOS and controls, while multiple regression analysis indicated SHBG as the main predicting factor of WBC. Finally, we picked out the group of overweight/obese (BMI $\geq 25 \mathrm{~kg} / \mathrm{m} 2$ ) women with PCOS and conducted another classification based on HOMA score (HOMA-IR $\leq 2$ : insulin-sensitive women, HOMA-IR $>2$ : insulin-resistant women) in the group of overweight and obese women with PCOS separately. In overweight women with PCOS, WBC, although higher in the group of insulin-resistant, did not differ significantly between the two groups, while in the subcategory of overweight women WBC was significantly $(p=0.02)$ higher
\end{abstract}


in the group of insulin-resistant women (HOMA-IR>2). CONCLUSIONS: Chronic low-grade inflammation and increased white cell count do occur in PCOS. Obesity and insulin resistance are the two leading parameters that act accumulatively in the development of leucocytosis, whereas hyperandrogenism does not seem to affect it.

Key words: Inflammation, Insulin resistance, Obesity, Polycystic ovary syndrome, White blood cells

\section{INTRODUCTION}

Polycystic ovary syndrome (PCOS) is the most common endocrinopathy in premenopausal women, with a prevalence of $6-9 \%$ according to NIH criteria, which rises to $15 \%$ according to the Rotterdam criteria. ${ }^{1-4}$ It is a heterogeneous disorder characterized by ovulatory dysfunction, clinical and biochemical hyperandrogenemia and polycystic ovaries and is associated with various metabolic abnormalities, including obesity, insulin resistance, metabolic syndrome, type 2 diabetes mellitus and dyslipidemia, thus increasing the lifelong risk for cardiovascular disease. ${ }^{5,6}$ While the pathogenesis of the syndrome remains uncertain, chronic low-grade inflammation is emerging as a new contributing factor, generatng new insights into the understanding of the syndrome.

Chronic low-grade inflammation has been demonstrated to promote atherosclerosis and create a favorable environment for atherothrombotic events. ${ }^{78}$ White blood cell count (WBC), CRP and others cytokines not only are involved in the process of atherosclerosis but have also been recognized as predictive markers of cardiovascular events. ${ }^{9-11}$ Oxidative stress and endothelial dysfunction are also closely related to chronic inflammation and seem to play a key role in the development of atherosclerotic plaques. ${ }^{12}$

Several experimental studies have disclosed the presence of subclinical inflammation in PCOS. Elevation of markers of chronic inflammation, such as WBC, CRP and IL-18, has been widely detected in women suffering from PCOS. ${ }^{13-16}$ As chronic inflammation is accompanied by endothelial inflammation, and consequently by endothelial dysfunction, concordant findings, with elevated levels of endothelin-1, plasminogen activator inhibitor-1 (PAI-1) and cellular adhesion molecules (CAMs), were also reported in women with PCOS. ${ }^{17-20}$ In addition to this, accumu- lating evidence supports the presence of oxidative stress and advanced glycation end-products (AGEs) in women with PCOS, implying the existence of a proatherogenic load in these women. ${ }^{21,22}$ Notably, increased serum AGES have been directly correlated with increased levels of endothelin-1 in women with PCOS. ${ }^{19}$ However, it has not as yet been clarified whether these inflammatory processes are associated with PCOS per se or are consequences of metabolic and anthropometric abnormalities, such as obesity, central accumulation of fat and insulin resistance that are commonly observed in PCOS.

The aim of the present study was to investigate the leucocyte count, a marker of subclinical inflammation, in women suffering from PCOS and compare these results with BMI and age-matched healthy women. Moreover, we examined whether WBC was correlated with anthropometric, metabolic and hormonal characteristics of the studied subjects in an attempt to investigate the role of low-grade chronic inflammation in the pathogenesis of PCOS.

\section{SUBJECTS AND METHODS}

\section{Subjects}

Two hundred and three (203) Greek Caucasian women with PCOS were recruited at the Eugenideio PCOS Clinic, University of Athens. All these women visited the PCOS Clinic complaining of menstrual abnormalities, hirsutism, acne and infertility.

Each patient with PCOS met the diagnostic criteria for PCOS based on the NIH criteria. Hyperandrogenemia was assessed as total testosterone levels above the 95th percentile of the levels detected in a group of normal menstruating women with normal cycles and, clinically, by the presence of hirsutism (Ferriman-Gallwey score $>8$ ) and acne. Chronic 
anovulation was assessed as oligomenorrhoea, i.e. less than eight cycles per year. Other hyperandrogenemic conditions, such as Cushing syndrome, late-onset 21-hydroxylase deficiency, androgen-secreting tumors and congenital adrenal hyperplasia, were ruled out. The Synacthene test was conducted in each woman with a basal 17-hydroxyprogesterone (17-OHP) plasma level $>1.5 \mathrm{ng} / \mathrm{ml}$ in the follicular phase of the menstrual cycle. In addition, seventy-six (76) age-matched, normoandrogenemic, regularly-menstruating women served as controls. The entire enrolled population was clinically healthy and not suffering from chronic or acute inflammatory diseases. They all had normal renal, hepatic and thyroid function and prolactin was within normal limits.

Among exclusion criteria were pregnancy, breastfeeding, known diabetes mellitus, hypertension or cardiovascular disease and intense physical activity. Oral contraceptives or other drugs that could interfere with the hormonal and metabolic studies, if administered, were discontinued for at least 3 months before the study. None of the patients had been on metformin therapy for more than six months before enrollment in the study. ${ }^{23}$ Regarding smoking, only 20 patients with PCOS smoked occasionally and they were asked to abstain from smoking during the study. Ovarian morphology was assessed in all subjects by transvaginal ultrasound and the Adams criteria were used to define it. ${ }^{24}$ Women with abnormal ovarian morphology in the control group were also excluded.

The study was approved by the Ethical Committee of the Medical School of the University of Athens and signed consent was obtained from all subjects after explanation of the nature and purpose of the study.

\footnotetext{
Assays

Body weight and height were measured. Body weight was measured using analogue scales, in light clothing, while height was measured barefoot using a stadiometer. Body mass index (BMI) was calculated to assess obesity. In order to evaluate insulin resistance, the homeostasis model assessment (HOMA) score was also calculated using the formula: fasting insulin $(\mathrm{mIU} / \mathrm{mL}) \mathrm{x}$ fasting glucose $(\mathrm{mmol} / \mathrm{L}) / 22.5$. Finally, the free androgen index (FAI) was calculated by the formula: testosterone (nmol/L)/SHBG (nmol/l) x100.
}

Blood samples were collected from all patients and healthy controls between 08.00 and $10.00 \mathrm{am}$, after an overnight fast. All samples were obtained during the early follicular phase of their menstrual cycle or at any time in anovulatory subjects with progesterone levels $<5 \mathrm{ng} / \mathrm{ml}$. Samples were centrifuged immediately and serum was stored at $-80{ }^{\circ} \mathrm{C}$ until assayed for glucose (GLU), insulin (INS), total testosterone (TT), sex hormone-binding globulin (SHBG), androstenedione (D4A), luteinizing hormone (LH), follicle stimulating hormone (FSH), 17-OHP, dehydroepiandrosterone sulfate (DHEA-S), E2, total cholesterol (TC), low-density lipoprotein (LDL), high-density lipoprotein (HDL), triglycerides (TGL), white blood count (WBC), alkaline phosphatase (ALP), serum glutamic oxaloacetic transaminase (SGOT), serum glutamic-pyruvic transaminase (SGPT).

Transvaginal ultrasound scans of the ovaries were performed during the follicular phase for ovulatory subjects and during the study period for the anovulatory subjects, confirmed with progesterone levels $<5$ $\mathrm{ng} / \mathrm{ml}$ as previously described. The Adams criteria were applied to define them.

\section{Statistics}

The statistical analysis was performed using Statistical Package for Social Sciences (SPSS) version 20 (SPSS, Chicago, IL, USA). $\mathrm{P}<0.05$ was considered to represent statistical significance. Continuous variables were assessed for normal distribution both graphically and by the Kolmogorov-Smirnov test. Variables with asymmetric distribution were analyzed by nonparametric statistical tests. Differences among continuous variables between the two groups were evaluated using the independent sample, two-tailed T-Test. Correlation between variables was evaluated by both Pearson's and Spearman's coefficients. Stepwise, partial correlation analyses controlling for BMI and age were also evaluated. Correlation between the variables was also assessed after adjusting all the laboratory and anthropometrical parameters for BMI. Multiple linear regression analysis was performed in the total population, setting white blood count as the independent variable. Finally, classifications based on BMI and HOMA score were conducted in order to assess more effectively these confounding factors. 


\section{RESULTS}

Anthropometric characteristics and the main hormonal and metabolic profile of PCOS patients and controls are presented in Table 1. All the continuous variables are expressed as mean $\pm \mathrm{SD}$.

In the total cohort $(\mathrm{N}=279)$, white blood count (WBC) was significantly higher $(\mathrm{p}=0.003)$ in the PCOS group compared with age-matched healthy women. In addition, WBC was positively correlated with BMI $(\mathrm{r}=0.461, \mathrm{p}<0.001)$, total testosterone $(\mathrm{r}=$ $0.210, \mathrm{p}<0.001)$, insulin $(\mathrm{r}=0.271, \mathrm{p}<0.001)$, triglycerides $(\mathrm{r}=0.285, \mathrm{p}<0.001)$, HOMA score $(\mathrm{r}=0.206$, $\mathrm{p}=0.001)$, FAI $(\mathrm{r}=0.329, \mathrm{p}<0.001)$ and negatively

Table 1. Anthropometric, hormonal and metabolic characteristics of PCOS patients and controls.

\begin{tabular}{|c|c|c|c|}
\hline & $\begin{array}{c}\text { PCOS } \\
(\mathrm{N}=203)\end{array}$ & $\begin{array}{c}\text { Controls } \\
(\mathrm{N}=76)\end{array}$ & $\mathbf{P}$ \\
\hline Age (years) & $25.26 \pm 6.24$ & $25.53 \pm 6.82$ & 0.754 \\
\hline BMI $\left(\mathrm{kg} / \mathrm{m}^{2}\right)$ & $27.89 \pm 7.73$ & $22.34 \pm 2.47$ & $<0.001$ \\
\hline $\mathrm{WBC}\left(\mathrm{x} 10^{9} / \mathrm{L}\right)$ & $7.04 \pm 1.6$ & $6.47 \pm 1.2$ & 0.003 \\
\hline $\begin{array}{l}\text { Total Testosterone } \\
(\mathrm{nmol} / \mathrm{L})\end{array}$ & $3.3 \pm 1.4$ & $2.08 \pm 0.55$ & $<0.001$ \\
\hline $\mathrm{D} 4 \mathrm{~A}(\mathrm{nmol} / \mathrm{L})$ & $12.39 \pm 5.72$ & $4.57 \pm 2.96$ & $<0.001$ \\
\hline DHEA-S (nmol/L) & $6543.6 \pm 4969.4$ & $7083.54 \pm 3365.3$ & 0.385 \\
\hline SHBG(nmol/L) & $37.78 \pm 21.89$ & $67.68 \pm 33.17$ & 0.010 \\
\hline FSH (IU/L) & $5.38 \pm 3.39$ & $4.93 \pm 2.56$ & 0.251 \\
\hline LH (IU/L) & $7.15 \pm 5.41$ & $7.80 \pm 7.89$ & 0.520 \\
\hline Insulin (pmol/l) & $95.21 \pm 63.82$ & $55.32 \pm 51.9$ & $<0.001$ \\
\hline Glucose $(\mathrm{mmol} / \mathrm{L})$ & $4.66 \pm 0.52$ & $4.84 \pm 0.51$ & 0.009 \\
\hline SGOT (U/L) & $19.11 \pm 7.12$ & $21.38 \pm 7.78$ & 0.033 \\
\hline SGPT(U/L) & $20.99 \pm 17.96$ & $27.66 \pm 9.07$ & 0.002 \\
\hline$\gamma \mathbf{G T}(\mathbf{U} / \mathbf{L})$ & $16.62 \pm 13.32$ & $16.42 \pm 11.14$ & 0.903 \\
\hline $\begin{array}{l}\text { Total cholesterol } \\
(\mathrm{mmol} / \mathrm{L})\end{array}$ & $4.58 \pm 0.84$ & $4.42 \pm 0.85$ & 0.185 \\
\hline $\mathrm{HDL}(\mathrm{mmol} / \mathrm{L})$ & $1.40 \pm 0.35$ & $1.59 \pm 0.33$ & $<0.001$ \\
\hline TGL (mmol/L) & $0.92 \pm 0.54$ & $0.8 \pm 0.29$ & 0.017 \\
\hline HOMA-IR & $2.80 \pm 2.11$ & $2.05 \pm 1.88$ & 0.007 \\
\hline FAI & $8.73 \pm 5.66$ & $3.07 \pm 1.65$ & 0.002 \\
\hline WHR & $0.82 \pm 0.08$ & & \\
\hline
\end{tabular}

Data are shown as mean \pm SD. $\mathrm{P}<0.05$ : statistical significance. WBC: white blood count, DHEA-S: dehydroepiandrosterone sulphate, SHBG: sex hormone-binding globulin, TGL: triglycerides, HOMA-IR: homeostasis model assessment, FAI: free androgen index. correlated with SHBG $(\mathrm{r}=-0.300, \mathrm{p}<0.001)$ and HDL $(\mathrm{r}=-0.222, \mathrm{p}<0.001)$. After controlling for BMI and age, WBC was significantly correlated only with SHBG $(r=-0.150, p=0.017)$ and triglycerides $(r=$ $0.193, \mathrm{p}=0.002$ ). Multiple regression analysis was carried out in the entire sample and data exhibited that BMI, TGL and SHBG were predictors of WBC (multicollinearity was not documented), but after adjusting for age, BMI and HOMA score, the statistically significant correlation was lost (Results not shown in the text).

It is widely acknowledged that abdominal obesity plays a unique role in the pathogenesis of low-grade inflammation. It is independently associated with insulin resistance and, therefore, subclinical inflammation. ${ }^{25,26}$ In our study, due to the fact that WHR was available only in the group of PCOS women, the role of central adiposity is assessed solely in this group. WBC correlated statistically significantly with WHR $(r=0,318, p<0.001)$ in PCOS women. In addition, multiple regression analysis in the PCOS group, including WHR, revealed BMI, SHBG and TGL as the main predicting factors of WBC. (Results are not presented). However, after adjusting for BMI, HOMA score and WHR, the correlations were lost and only TGL was statistically significantly $(r=0.186, p=0.017)$ correlated with WBC. Finally, multinomial logistic regression analysis in the group of PCOS women was conducted. Overweight/obesity was the only independent risk factor for elevated WBC (higher tertile) (OR:0.907 CI:0.85-0.96, p=0.002).

In order to eliminate the confounding effect of BMI, the anthropometric and laboratory data of both groups were adjusted for BMI. WBC was significantly higher in the PCOS group even after the adjustment and was still correlated with SHBG $(\mathrm{P}=0.017)$, triglycerides $(\mathrm{P}=0.002)$ and marginally with FAI $(\mathrm{P}=0.061)$ (Table 2).

Subsequently, after dividing the whole sample into two groups, based on BMI (Lean: BMI $<25 \mathrm{~kg} / \mathrm{m}^{2}$ and Overweight/obese: $\mathrm{BMI} \geq 25 \mathrm{~kg} / \mathrm{m}^{2}$ ), we focused our analysis on the lean population (both controls and women with PCOS) in order to investigate WBC in normal-weight, non-insulin-resistant women and assess the role of obesity in subclinical inflammation (Table 3). In the lean subgroups, WBC did not differ 
Table 2. Impact of different anthropometric and metabolic characteristics on WBC before an d after adjusting for BMI in the total cohort.

\begin{tabular}{lccccc}
\hline & \multicolumn{2}{c}{$\begin{array}{c}\text { Before BMI } \\
\text { Adjustment }\end{array}$} & & \multicolumn{2}{c}{$\begin{array}{c}\text { After BMI } \\
\text { adjustment }\end{array}$} \\
\cline { 2 - 3 } \cline { 5 - 6 } Independent variable & $\mathbf{R}$ & $\mathbf{P}$ & & $\mathbf{R}$ & $\mathbf{P}$ \\
\hline Total testosterone & 0.210 & 0.001 & & 0.048 & 0.423 \\
SHBG & -0.300 & $<0.001$ & & -0.150 & $\mathbf{0 . 0 1 7}$ \\
Insulin & 0.271 & $<0.001$ & & 0.057 & 0.381 \\
HDL & -0.220 & $<0.001$ & & -0.078 & 0.217 \\
TGL & 0.285 & $<0.001$ & & 0.193 & $\mathbf{0 . 0 0 2}$ \\
HOMA-IR & 0.206 & 0.001 & & 0.019 & 0.762 \\
FAI & 0.329 & $<0.001$ & & 0.119 & $\mathbf{0 . 0 6 1}$ \\
PCOS status & 0.119 & 0.047 & & 0.01 & 0.856 \\
\hline
\end{tabular}

significantly between PCOS patients and controls and was positively correlated with age $(\mathrm{R}=0.162$, $\mathrm{p}=0.030)$, BMI $(\mathrm{R}=0.205, \mathrm{p}=0.009)$, insulin $(\mathrm{R}=$ $0.174, p=0.030)$ and negatively correlated with SHBG $(\mathrm{R}=-0.257, \mathrm{p}=0.002)$. Finally, multiple regression analysis in the whole lean population indicated SHBG as the main predicting factor of WBC. Regression analysis, including PCOS status as a variable, led to the same results. (Results are not presented).

In order to evaluate the contribution of insulin resistance to low-grade inflammation in PCOS, we picked out the group of overweight/obese (BMI $\geq 25$ $\mathrm{kg} / \mathrm{m}^{2}$ ) women with PCOS and conducted another classification based on HOMA score (HOMA-IR $\leq 2$ : insulin-sensitive women, HOMA-IR $>2$ : insulin-resistant women) (Tables 4, 5) in the group of overweight and obese women with PCOS separately. Regarding overweight $\left(25<\mathrm{BMI} \leq 30 \mathrm{~kg} / \mathrm{m}^{2}\right)$ women with PCOS, the two groups that were formed were BMI-matched, age-matched and WHR-matched. WBC, although higher in the group of insulin-resistant, did not differ significantly between the two groups. Furthermore, WBC correlated statistically significantly only with TGL $(r=0.359, p=0.040)$ and FAI $(r=0.393, p=0.024)$. Finally, we ran a multiple regression analysis model, which pointed to SHBG as the only predicting factor of WBC. (Results are not presented). On the other

Table 3. Anthropometric, hormonal and metabolic characteristics of PCOS patients and controls of the lean subgroup $\left(\mathrm{BMI}<25 \mathrm{~kg} / \mathrm{m}^{2}\right)$.

\begin{tabular}{|c|c|c|c|}
\hline & \multicolumn{2}{|c|}{$\mathrm{BMI}<25 \mathrm{~kg} / \mathrm{m}^{2}$} & \multirow[b]{2}{*}{$\mathbf{P}$} \\
\hline & $\operatorname{PCOS}(\mathrm{N}=110)$ & Controls $(\mathrm{N}=64)$ & \\
\hline Age (years) & $24.04 \pm 5.73$ & $25.05 \pm 6.88$ & 0.286 \\
\hline $\mathrm{BMI}\left(\mathrm{kg} / \mathrm{m}^{2}\right)$ & $21.74 \pm 2.59$ & $21.55 \pm 1.74$ & 0.563 \\
\hline $\mathrm{WBC}\left(\mathrm{x} 10^{9} / \mathrm{L}\right)$ & $6.39 \pm 1.3$ & $6.5 \pm 1.2$ & 0.535 \\
\hline Total Testosterone (nmol/L) & $3.1 \pm 1.20$ & $2.04 \pm 0.55$ & $<0.001$ \\
\hline $\mathrm{D} 4 \mathrm{~A}(\mathrm{nmol} / \mathrm{L})$ & $11.97 \pm 4.36$ & $4.78 \pm 3$ & $<0.001$ \\
\hline DHEAS (nmol/L) & $6733.5 \pm 5436.5$ & $6812 \pm 3365.3$ & 0.919 \\
\hline SHBG(nmol/L) & $46.10 \pm 21.53$ & $69.94 \pm 34.94$ & 0.001 \\
\hline FSH (IU/L) & $5.02 \pm 2.23$ & $4.90 \pm 2.66$ & 0.773 \\
\hline LH (IU/L) & $7.11 \pm 5.44$ & $8 \pm 8.38$ & 0.467 \\
\hline Insulin (pmol/l) & $68.6 \pm 30.83$ & $56.76 \pm 23.87$ & 0.732 \\
\hline Glucose $(\mathrm{mmol} / \mathrm{L})$ & $4.52 \pm 0.42$ & $4.83 \pm 0.52$ & $<0.001$ \\
\hline SGOT (U/L) & $17.47 \pm 4.85$ & $21.64 \pm 7.88$ & $<0.001$ \\
\hline SGPT(U/L) & $15.02 \pm 7.02$ & $27.56 \pm 9.71$ & $<0.001$ \\
\hline$\gamma \mathbf{G T}(\mathbf{U} / \mathbf{L})$ & $11.13 \pm 3.61$ & $16.85 \pm 12.09$ & $<0.001$ \\
\hline Total cholesterol $(\mathrm{mmol} / \mathrm{L})$ & $4.44 \pm 0.80$ & $4.35 \pm 0.79$ & 0.715 \\
\hline $\mathrm{HDL}(\mathrm{mmol} / \mathrm{L})$ & $1.52 \pm 0.35$ & $1.6 \pm 0.35$ & 0.189 \\
\hline TGL (mmol/L) & $0.75 \pm 0.33$ & $0.8 \pm 0.29$ & 0.319 \\
\hline HOMA-IR & $1.97 \pm 0.96$ & $2.06 \pm 1.85$ & 0.755 \\
\hline FAI & $6.72 \pm 5.55$ & $2.92 \pm 1.54$ & $<0.001$ \\
\hline
\end{tabular}


Table 4. Anthropometric and laboratory data of overweight/obese $\left(25<\mathrm{BMI} \leq 30 \mathrm{~kg} / \mathrm{m}^{2}\right)$ women with PCOS, classified in two categories based on HOMA Score (HOMA-IR $\leq 2$ : insulin-sensitive women, HOMA-IR $>2$ : insulin-resistant women)

\begin{tabular}{|c|c|c|c|}
\hline & \multicolumn{2}{|c|}{ PCOS $-25<\mathrm{BMI} \leq 30 \mathrm{~kg} / \mathrm{m}^{2}$} & \multirow[b]{2}{*}{$\mathbf{P}$} \\
\hline & Insulin Sensitive $(\mathbf{N}=13)$ & Insulin Resistant $(\mathrm{N}=18)$ & \\
\hline Age (years) & $24.92 \pm 5.70$ & $22.28 \pm 5.88$ & 0.220 \\
\hline BMI $\left(\mathrm{kg} / \mathrm{m}^{2}\right)$ & $26.85 \pm 1.36$ & $27.35 \pm 1.32$ & 0.315 \\
\hline $\mathrm{WBC}\left(\times 10^{9} / \mathrm{L}\right)$ & $6.71 \pm 1.44$ & $7.03 \pm 1.52$ & 0.565 \\
\hline WHR & $0.83 \pm 0.09$ & $0.81 \pm 0.05$ & 0.386 \\
\hline Total Testosterone $(\mathrm{nmol} / \mathrm{L})$ & $2.8 \pm 1.2$ & $3.9 \pm 1.4$ & 0.028 \\
\hline $\mathrm{D} 4 \mathrm{~A}(\mathrm{nmol} / \mathrm{L})$ & $13.33 \pm 4.74$ & $11.93 \pm 5.51$ & 0.476 \\
\hline DHEAS (nmol/L) & $5778 \pm 2646$ & $6318 \pm 3450$ & 0.628 \\
\hline SHBG(nmol/L) & $35.36 \pm 11.90$ & $36.75 \pm 32.27$ & 0.875 \\
\hline $\mathrm{FSH}(\mathrm{mIU} / \mathrm{ml})$ & $5.01 \pm 1.72$ & $4.64 \pm 1.35$ & 0.516 \\
\hline LH (mIU/ml) & $8.86 \pm 1.09$ & $6.97 \pm 4.68$ & 0.525 \\
\hline Insulin (pmol/L) & $50.55 \pm 20.27$ & $113.62 \pm 56.11$ & $<0.001$ \\
\hline Glucose $(\mathrm{mmol} / \mathrm{L})$ & $4.44 \pm 0.4$ & $4.61 \pm 0.38$ & 0.245 \\
\hline SGOT (U/L) & $17.62 \pm 3.68$ & $16.56 \pm 3.16$ & 0.398 \\
\hline SGPT(U/L) & $15.31 \pm 5.96$ & $17.50 \pm 7.50$ & 0.373 \\
\hline$\gamma \mathrm{GT}(\mathrm{U} / \mathrm{L})$ & $14.08 \pm 7.55$ & $17.24 \pm 18.68$ & 0.588 \\
\hline Total cholesterol $(\mathrm{mmol} / \mathrm{L})$ & $4.31 \pm 0.63$ & $4.31 \pm 0.70$ & 0.977 \\
\hline $\mathrm{HDL}(\mathrm{mmol} / \mathrm{L})$ & $1.45 \pm 0.42$ & $1.40 \pm 0.40$ & 0.761 \\
\hline TGL (mmol/L) & $0.73 \pm 0.28$ & $0.76 \pm 0.30$ & 0.805 \\
\hline FAI & $2.66 \pm 1.6$ & $4.95 \pm 3.25$ & 0.021 \\
\hline
\end{tabular}

Table 5. Anthropometric and laboratory data of overweight/obese (BMI $\geq 30 \mathrm{~kg} / \mathrm{m}^{2}$ ) women with PCOS, classified in two categories based on HOMA Score (HOMA-IR $\leq 2$ : insulin-sensitive women, HOMA-IR $>2$ : insulin-resistant women)

\begin{tabular}{|c|c|c|c|}
\hline & \multicolumn{2}{|c|}{$\mathrm{PCOS}-\mathrm{BMI} \geq 30 \mathrm{~kg} / \mathrm{m}^{2}$} & \multirow[b]{2}{*}{$\mathbf{P}$} \\
\hline & Insulin Sensitive $(\mathrm{N}=12)$ & Insulin Resistant $(\mathrm{N}=57)$ & \\
\hline Age (years) & $23.42 \pm 5.01$ & $28.39 \pm 6.71$ & 0.018 \\
\hline BMI $\left(\mathrm{kg} / \mathrm{m}^{2}\right)$ & $34.65 \pm 5.30$ & $37.07 \pm 5.60$ & 0.175 \\
\hline $\mathrm{WBC}\left(\mathrm{x} 10^{9} / \mathrm{L}\right)$ & $7.78 \pm 1.8$ & $8.48 \pm 1.8$ & 0.020 \\
\hline WHR & $0.86 \pm 0.07$ & $0.89 \pm 0.08$ & 0.270 \\
\hline Total Testosterone (nmol/L) & $3.6 \pm 1.6$ & $3.6 \pm 1.7$ & 0.990 \\
\hline $\mathrm{D} 4 \mathrm{~A}(\mathrm{nmol} / \mathrm{L})$ & $15.56 \pm 10.95$ & $12.04 \pm 5.33$ & 0.256 \\
\hline DHEAS (nmol/L) & $8277.7 \pm 6947.8$ & $5807.9 \pm 3066.8$ & 0.087 \\
\hline SHBG (nmol/L) & $29.46 \pm 15.02$ & $26.66 \pm 15.33$ & 0.124 \\
\hline $\mathrm{FSH}(\mathrm{mIU} / \mathrm{ml})$ & $5.70 \pm 1.73$ & $5.53 \pm 3.46$ & 0.882 \\
\hline $\mathrm{LH}(\mathrm{mIU} / \mathrm{ml})$ & $6.99 \pm 3.64$ & $6.79 \pm 3.85$ & 0.878 \\
\hline Insulin $(\mathrm{pmol} / \mathrm{L})$ & $42.72 \pm 17.04$ & $113.64 \pm 47.88$ & $<0.001$ \\
\hline Glucose $(\mathrm{mmol} / \mathrm{L})$ & $4.72 \pm 0.79$ & $4.90 \pm 0.56$ & 0.354 \\
\hline SGOT (U/L) & $21.42 \pm 7.78$ & $22.04 \pm 9.80$ & 0.733 \\
\hline SGPT(U/L) & $33.42 \pm 26$ & $29.18 \pm 23.06$ & 0.605 \\
\hline$\gamma \mathrm{GT}(\mathrm{U} / \mathrm{L})$ & $20 \pm 7.55$ & $24.24 \pm 11.44$ & 0.193 \\
\hline Total cholesterol (mmol/L) & $4.96 \pm 0.65$ & $4.88 \pm 0.90$ & 0.760 \\
\hline $\mathrm{HDL}(\mathrm{mmol} / \mathrm{L})$ & $1.26 \pm 0.19$ & $1.19 \pm 0.25$ & 0.429 \\
\hline TGL (mmol/L) & $1.20 \pm 0.92$ & $1.16 \pm 0.5$ & 0.855 \\
\hline FAI & $12.21 \pm 8.35$ & $13.50 \pm 8.42$ & 0.267 \\
\hline
\end{tabular}


hand, in the subcategory of obese women with PCOS, the two groups that originate from HOMA classification were BMI-matched and WHR-matched, but not age-matched. WBC was significantly $(\mathrm{p}=0.02)$ higher in the group of insulin-resistant women (HOMAIR $>2$ ). Meanwhile, WBC was positively correlated with BMI $(\mathrm{R}=0.354, \mathrm{p}=0.002)$ and triglycerides $(\mathrm{R}=0.218, \mathrm{p}=0.04)$ and marginally negatively correlated with HDL $(\mathrm{R}=-0.222, \mathrm{p}=0.058)$.

To summarize, Figure 1 illustrates very clearly the variance of WBC in the different subgroups that we studied, depicting in a very comprehensive way the effect of the two catalytic factors, BMI and insulin resistance on WBC and low-grade inflammation in general.

\section{DISCUSSION}

These data clearly suggest that chronic, low-grade inflammation and, particularly, elevated white blood count do occur in PCOS. More specifically, women with PCOS had statistically significantly higher WBC in comparison with their normal-ovulating, nonhyperandrogenic, age-matched peers, mainly as a result of the synergistic effect of obesity and insulin resistance and not due to PCOS per se.

PCOS is one of the most common reproductive disorders, with a heterogeneous clinical profile, which includes, apart from the hormonal dysfunction, metabolic aberrations. It has even been suggested that PCOS represents a female subtype of metabolic syndrome, carrying a potential proatherogenic load. ${ }^{27}$ Although the pathogenesis of the syndrome has not yet been clarified, low-grade inflammation is gradually being established as a pathogenetic factor.

There are a plethora of relevant papers in the literature regarding the role of $\mathrm{WBC}$ in the pathogenesis of PCOS. WBC was ubiquitously demonstrated to be elevated in women suffering from PCOS, in comparison with normal, healthy women. The crucial question that remains to be answered is if this leucocytosis is an innate characteristic of the syndrome or an aftermath of its metabolic aspects. ${ }^{15,28,29}$ In our cohort of 203 women with PCOS (NIH Criteria) and 76 healthy, age-matched women, WBC was significantly correlated with BMI and various markers of insulin resistance. In addition to the above results, which demonstrate the catalytic role of BMI in low-grade inflammation, BMI was seen to be one of the main predicting factors of WBC when we applied multiple regression analysis in the whole sample. Another important predictive factor of WBC that was shown in multiple regression analysis was SHBG, which is commonly known to be a predictive marker not only for insulin resistance but also for diabetes type II and hypertension. ${ }^{30,31}$ The above results are in concord-

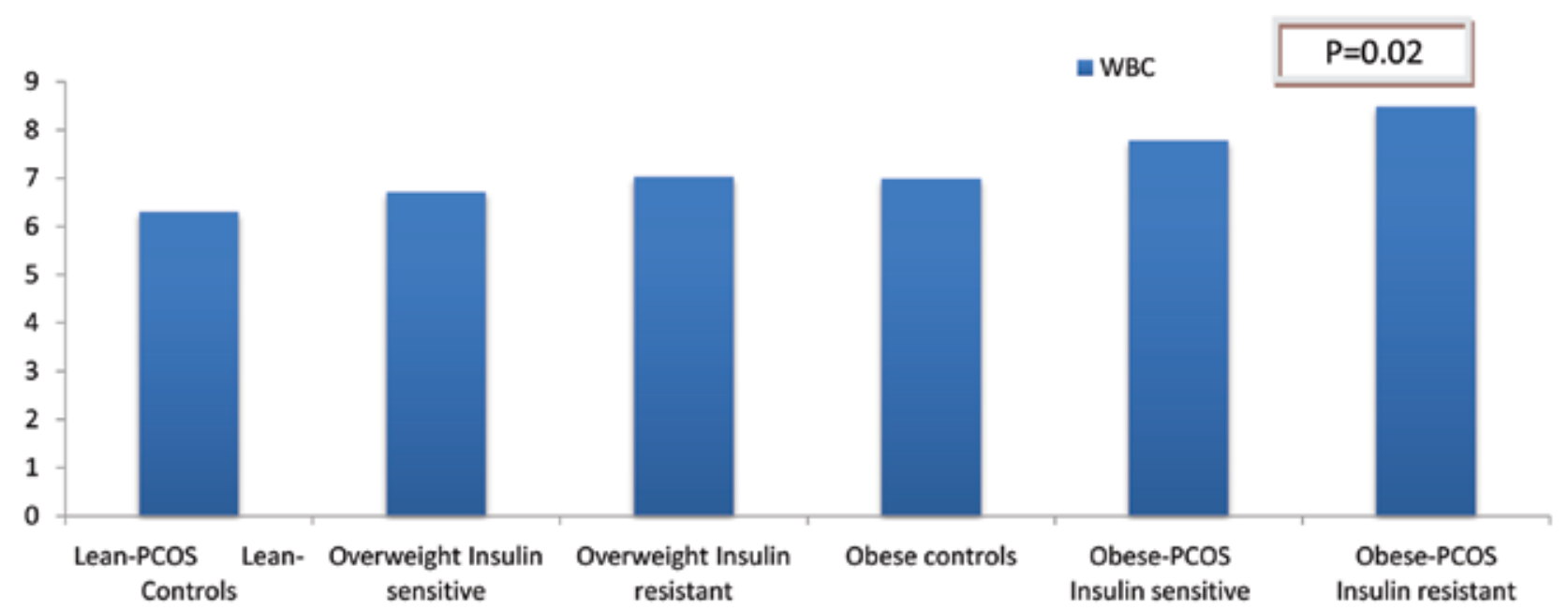

Figure 1. WBC in the different subgroups studied, depicting the synergistic role of obesity and insulin resistance in low-grade inflammation in PCOS. The only statistically significant difference was observed between the subgroups based on Insulin sensitivity. $(\mathrm{p}=0.02)$. 
ance with the majority of the current literature, which reveals obesity and insulin resistance as the main factors responsible for the elevation of WBC in PCOS. More specifically, BMI and the HOMA score appear to be the most important predicting factors of white cell count in women with PCOS. ${ }^{15,32,33}$ As a result, insulin resistance is highlighted as the main culprit of elevated WBC in PCOS, which is also supported by the fact that metformin was shown to reduce WBC after a 6-month administration. ${ }^{23}$

By contrast, in our study androgens, FAI and PCOS status failed to display any statistically significant correlation with WBC. What is more, PCOS status failed to predict $\mathrm{WBC}$ in the whole population, after adjusting for age, BMI and HOMA score. The above results in conjunction with the fact that WBC did not differ significantly between PCOS and controls, when only lean subjects were included, led us to the conclusion that hyperandrogenemia is excluded from the equation of leucocytosis. In contrast to our results, Phelan et al. showed that although low grade inflammation in women with PCOS was closely related to insulin resistance, WBC was elevated in women with PCOS, even when insulin resistance was adjusted, suggesting that leucocytosis is an innate characteristic of PCOS. ${ }^{13}$

It is widely acknowledged that obesity and insulin resistance are closely linked to low-grade inflammation. Thus, in order to evaluate the contribution of these fundamental factors to the inflammatory state observed in PCOS, we examined each one of them separately.

Firstly, after adjusting the whole sample for BMI, we observed that WBC remained higher in women suffering from PCOS and WBC was still correlated significantly with SHBG, triglycerides and marginally with FAI. Moreover, we dichotomized our sample into two groups based on BMI in order to focus our analysis in the lean (BMI $<25 \mathrm{~kg} / \mathrm{m}^{2}$ ), non-insulin resistant population. In this subgroup, WBC did not differ significantly between women with PCOS and age-matched controls. The above outcomes underscore the fact that in the absence of obesity and insulin resistance, WBC displays no variance between healthy women and women with PCOS. As a result, elevated WBC cannot be attributed to PCOS per se.
Secondly, in order to assess further and more deeply the role of insulin resistance and its interaction with obesity, we conducted another classification separately in the group of overweight $(25<\mathrm{BMI} \leq 30 \mathrm{~kg} /$ $\left.\mathrm{m}^{2}\right)$ and obese $\left(\mathrm{BMI} \geq 30 \mathrm{~kg} / \mathrm{m}^{2}\right)$ women with PCOS, based on the HOMA score. WBC was significantly higher in the group of obese insulin-resistant women (HOMA-IR $>2$ ) with PCOS. All these data lead to the conclusion that obesity and insulin resistance act synergistically in the induction of low-grade inflammation, a fact that is depicted clearly in the histogram provided below (Figure 1).

Another issue worth mentioning is the strong association of elevated triglycerides and low HDL levels with increased WBC before and after adjusting for BMI, a fact that is also demonstrated in in vitro models, which reveal novel triglyceride-mediated activation of neutrophils. ${ }^{34}$ Finally, it was surprising to find that fasting glucose plasma levels, as well as markers of hepatic function (SGOT, SGPT) were statistically significantly higher in controls. The exact reverse effect was expected, as impaired glucose homeostasis and non-alcoholic fatty liver disease are metabolic sequelae of PCOS.

\section{Major limitations of the study}

Among the major limitations of our study was that the role of central fat accumulation in low grade inflammation was not clearly evaluated. It is widely known that central fat excess is strongly associated with an increase in inflammatory markers and may be involved in the generation of chronic inflammation. ${ }^{35}$ Furthermore, Puder et al. demonstrated that central fat excess, rather than PCOS per se, is the main cause of subclinical inflammation in women with PCOS. ${ }^{33}$ Unfortunately, WHR was available only in the women suffering from PCOS. The absence of analogous measurements in the group of controls was a substantial obstacle in our effort to obtain a deeper understanding of the role of abdominal adiposity in low-grade inflammation. In addition, no other inflammatory markers (e.g. CRP, ESR, Fibrinogen) were measured. Finally, another limitation of our study is that the exercise intensity was not evaluated in our population, as exercise attenuates insulin resistance and improves inflammatory pattern in women with PCOS. ${ }^{36}$ 
In conclusion, $\mathrm{WBC}$, which is a potent predictor of cardiovascular disease, is elevated in women with PCOS. Obesity and insulin resistance, and not PCOS per se, seem to play the key role in this inflammatory state. These two leading parameters act accumulatively in the development of leucocytosis. However, further investigation is required in order to elucidate the mechanisms linking PCOS with inflammation and the possible clinical implications of these findings. In addition, long-term investigation is necessary so as to assess the role of low-grade, chronic inflammation as a potential predictive marker of increased risk for cardiovascular disease in women suffering from PCOS.

\section{REFERENCES}

1. Diamanti-Kandarakis E, Kouli CR, Bergiele AT, et al, A survey of the polycystic ovary syndrome in the Greek island of Lesbos: hormonal and metabolic profile. 1999 J Clin Endocrinol Metab 84: 4006-4011.

2. Azziz R, Dumesic DA, Goodarzi MO, 2011 Polycystic ovary syndrome: an ancient disorder? Fertil Steril 95: 1544-1548.

3. Azziz R, Woods KS, Reyna R, Key TJ, Knochenhauer ES, Yildiz BO, 2004 The prevalence and features of the polycystic ovary syndrome in an unselected population. J Clin Endocrinol Metab 89: 2745-2749.

4. March WA, Moore VM, Willson KJ, Phillips DI, Norman RJ, Davies MJ, 2010 The prevalence of polycystic ovary syndrome in a community sample assessed under contrasting diagnostic criteria. Hum Reprod 25: 544551.

5. Azziz R, Carmina E, Dewailly D, et al, 2006 Positions statement: criteria for defining polycystic ovary syndrome as a predominantly hyperandrogenic syndrome: an Androgen Excess Society guideline. J Clin Endocrinol Metab 91: 4237-4245.

6. Diamanti-Kandarakis E, Dunaif A, 2012 Insulin resistance and the polycystic ovary syndrome revisited: an update on mechanisms and implications. Endocr Rev 33: 981-1030.

7. Libby P, Theroux P, 2005 Pathophysiology of coronary artery disease. Circulation 111: 3481-3488.

8 . Libby P, 2002 Inflammation in atherosclerosis. Nature 420: $868-874$

9. Kannel WB, Anderson K, Wilson PW, 1992 White blood cell count and cardiovascular disease. Insights from the Framingham Study. JAMA 267: 1253-1256.

10. Tedgui A, Mallat Z, 2006 Cytokines in atherosclerosis: pathogenic and regulatory pathways. Physiol Rev 86: 515-581.

11. Jialal I, Devaraj S, 2001 Inflammation and atherosclerosis: the value of the high-sensitivity $\mathrm{C}$-reactive protein assay as a risk marker. Am J Clin Pathol 116: Suppl 1: 108-115.

12. Hulsmans M, Holvoet P, 2010 The vicious circle between oxidative stress and inflammation in atherosclerosis. $\mathrm{J}$ Cell Mol Med 14: 70-78.

13. Phelan N, O'Connor A, Kyaw Tun T, et al, 2013 Leucocytosis in women with polycystic ovary syndrome (PCOS) is incompletely explained by obesity and insulin resistance. Clin Endocrinol 78: 107-113.

14. Escobar-Morreale HF, Luque-Ramirez M, Gonzalez F, 2011 Circulating inflammatory markers in polycystic ovary syndrome: a systematic review and metaanalysis. Fertil Steril 95: 1048-1058.

15. Orio F Jr., Palomba S, Cascella T, et al, 2005 The increase of leukocytes as a new putative marker of low-grade chronic inflammation and early cardiovascular risk in polycystic ovary syndrome J Clin Endocrinol Metab 90: 2-5.

16. Escobar-Morreale HF, Botella-Carretero JI, Villuendas G, Sancho J, San Millán JL, 2004 Serum interleukin-18 concentrations are increased in the polycystic ovary syndrome: relationship to insulin resistance and to obesity. J Clin Endocrinol Metab 89: 806-811.

17. Tarkun I, Arslan BC, Canturk Z, Türemen E, Sahin T, Duman C, 2004 Endothelial dysfunction in young women with polycystic ovary syndrome: relationship with insulin resistance and low-grade chronic inflammation. J Clin Endocrinol Metab 89: 5592-5596.

18. Diamanti-Kandarakis E, Paterakis T, Kandarakis HA, 2006 Indices of low-grade inflammation in polycystic ovary syndrome. Ann N Y Acad Sci 1092: 175-186.

19. Christakou C, Economou F, Livadas S, et al, 2011 Strong and positive association of endothelin-1 with AGEs in PCOS: a causal relationship or a bystander? Hormones (Athens) 10: 292-297.

20. Diamanti-Kandarakis E, Palioniko G, Alexandraki K, Bergiele A, Koutsouba T, Bartzis M, 2004 The prevalence of $4 \mathrm{G} 5 \mathrm{G}$ polymorphism of plasminogen activator inhibitor-1 (PAI-1) gene in polycystic ovarian syndrome and its association with plasma PAI-1 levels. Eur J Endocrinol 150: 793-798.

21. Diamanti-Kandarakis E, Piperi C, Kalofoutis A, Creatsas G, 2005 Increased levels of serum advanced glycation end-products in women with polycystic ovary syndrome. Clin Endocrinol 62: 37-43.

22. Diamanti-Kandarakis E, Piouka A, Livadas S, et al, 2009 Anti-mullerian hormone is associated with advanced glycosylated end products in lean women with polycystic ovary syndrome. Eur J Endocrinol 160: 847-853.

23. Orio F, Manguso F, Di Biase S, et al, 2007 Metformin administration improves leukocyte count in women with polycystic ovary syndrome: a 6-month prospective study. Eur J Endocrinol 157: 69-73.

24. Adams J, Franks S, Polson DW, et al, 1985 Multifollicular ovaries: clinical and endocrine features and 
response to pulsatile gonadotropin releasing hormone. Lancet 2: 1375-1379.

25. Sathyapalan T, Atkin S, 2012 Recent advances in cardiovascular aspects of polycystic ovary syndrome. Eur J Endocrinol 166: 575-583.

26. Duleba AJ, Dokras, 2012 Is PCOS an inflammatory process? Fertil Steril 97: 7-12.

27. Sam S, Dunaif A, 2003 Polycystic ovary syndrome: syndrome XX? Trends Endocrinol Metab 14: 365-370.

28. Herlihy AC, Kelly RE, Hogan JL, O’Connor N, Farah N, Turner MJ, 2011 Polycystic ovary syndrome and the peripheral blood white cell count. J Obstet Gynaecol 31: 242-244.

29. Shi Y, Han T, Cui L, et al, 2013 White blood cell differential counts in patients with polycystic ovary syndrome: a pilot study on Chinese women. Eur J Obstet Gynecol Reprod Biol 170: 162-164.

30. Kajaia N, Binder H, Dittrich R, et al, 2007 Low sex hormone-binding globulin as a predictive marker for insulin resistance in women with hyperandrogenic syndrome. Eur J Endocrinol 157: 499-507.

31. Ding EL, Song Y, Manson JE, et al, 2009 Sex hormone- binding globulin and risk of type 2 diabetes in women and men. N Engl J Med 361: 1152-1163.

32. Ibanez L, Jaramillo AM, Ferrer A, de Zegher F, 2005 High neutrophil count in girls and women with hyperinsulinaemic hyperandrogenism: normalization with metformin and flutamide overcomes the aggravation by oral contraception. Hum Reprod 20: 2457-2462.

33. Puder JJ, Varga S, Kraenzlin M, De Geyter C, Keller U, Müller B, 2005 Central fat excess in polycystic ovary syndrome: relation to low-grade inflammation and insulin resistance. J Clin Endocrinol Metab 90: 6014-6021.

34. Alipour A, van Oostrom AJ, Izraeljan A, et al, 2008 Leukocyte activation by triglyceride-rich lipoproteins. Arterioscler Thromb Vasc Biol 28: 792-797.

35. Wajchenberg BL, 2000 Subcutaneous and visceral adipose tissue: their relation to the metabolic syndrome. Endocr Rev 21: 697-738.

36. Giallauria F, Palomba S, Maresca L, et al, 2008 Exercise training improves autonomic function and inflammatory pattern in women with polycystic ovary syndrome (PCOS). Clin Endocrinol 69: 792-798. 